\title{
Artefacts in 1.5 Tesla and 3 Tesla cardiovascular magnetic resonance imaging in patients with leadless cardiac pacemakers
}

Daniel Kiblboeck ${ }^{1 *}$, Christian Reiter ${ }^{1}$, Juergen Kammler ${ }^{1}$, Pierre Schmit ${ }^{2}$, Hermann Blessberger $^{1}$, Joerg Kellermair ${ }^{1}$, Franz Fellner ${ }^{2,3}$ and Clemens Steinwender ${ }^{1,4}$

\begin{abstract}
Background: There are limited data on patients with leadless cardiac pacemakers (LCP) undergoing magnetic resonance imaging. The aim of this prospective, single-center, observational study was to evaluate artefacts on cardiovascular magnetic resonance (CMR) images in patients with LCP.

Methods: Fifteen patients with Micra ${ }^{\mathrm{TM}}$ LCP, implanted at least 6 weeks prior to CMR scan, were enrolled and underwent either 1.5 Tesla or 3 Tesla CMR imaging. Artefacts were categorized into grade 1 (excellent image quality), grade 2 (good), grade 3 (poor) and grade 4 (non-diagnostic) for each myocardial segment. One patient was excluded because of an incomplete CMR investigation due to claustrophobia.

Results: LCP caused an arc-shaped artefact $\left(0.99 \pm 0.16 \mathrm{~cm}^{2}\right)$ at the right ventricular (RV) apex. Of 224 analyzed myocardial segments of the left ventricle (LV) 158 (70.5\%) were affected by grade 1, 27 (12.1\%) by grade 2, 17 (7.6\%) by grade 3 and 22 (9.8\%) by grade 4 artefacts. The artefact burden of grade 3 and 4 artefacts was significantly higher in the 3 Tesla group (3 Tesla vs 1.5 Tesla: $3.7 \pm 1.6$ vs $1.9 \pm 1.4$ myocardial segments per patient, $p=0.03$ ). A high artefact burden was particularly observed in the mid anteroseptal, inferoseptal and apical septal myocardial segments of the LV and in the mid and apical segments of the RV. Quantification of LV function and assessment of valves were feasible in all patients. We did not observe any clinical or device-related adverse events.

Conclusion: CMR imaging in patients with LCP is feasible with excellent to good image quality in the majority of LV segments. The artefact burden is comparable small allowing an accurate evaluation of LV function, cardiac structures and valves. However, artefacts in the mid anteroseptal, inferoseptal and apical septal myocardial segments of the LV due to the LCP may impair or even exclude diagnostic evaluation of these segments. Artefacts on CMR images may be reduced by the use of 1.5 Tesla CMR scanners.
\end{abstract}

Keywords: Artefacts, Leadless cardiac pacemakers, Cardiovascular magnetic resonance imaging, 1.5 Tesla, 3 Tesla

\footnotetext{
* Correspondence: daniel.kiblboeck@kepleruniklinikum.at

'Department of Cardiology, Kepler University Hospital Linz, Med Campus III,

Krankenhausstraße 9, 4021 Linz, Austria

Full list of author information is available at the end of the article
}

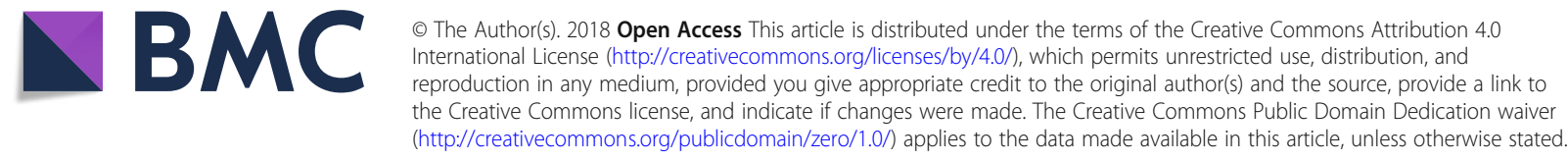




\section{Background}

Cardiovascular magnetic resonance (CMR) imaging, which has become a versatile, non-invasive imaging tool, allows a comprehensive evaluation of patients with cardiovascular diseases [1]. The different CMR imaging sequences offer the assessment of myocardial function, wall motion abnormalities, viability, coronary perfusion, valves and tissue characterization [1]. Potential hazards for patients with conventional cardiac pacemakers undergoing magnetic resonance imaging (MRI) are radiofrequency-induced heating of lead tips, pacing dysfunction and changes in capture threshold [2]. Several studies have demonstrated safety and feasibility of MRI conditional cardiac pacemakers and implantable cardioverter defibrillators (ICD) [3-11]. Leadless cardiac pacemaker (LCP) therapy was recently introduced clinically to overcome complications in transvenous pacemaker therapy, such as lead dislogdement and perforation with pericardial effusion, pocket hematoma and device infections [12-14]. The Micra ${ }^{\text {тм }}$ LCP (Medtronic, Minneapolis, USA), which was investigated in this study, is a MRI conditional cardiac, single chamber pacemaker. The device sizes of $25.9 \times 6.7 \mathrm{~mm}$ with an integrated lithium silver vanadium oxide, carbon monofluoride battery covered in titanium and is fixed with self-expanding nitinol tines in the right ventricle (RV) [14].

Pacemakers cause metallic susceptibility artefacts due to distortion of the magnetic field [15]. There are limited data about patients with LCP undergoing CMR imaging [16]. To the best of our knowledge, there are no prospective studies in the literature about artefacts on CMR imaging in patients with implanted LCP. It is unknown, whether CMR imaging provides best image quality or less artefacts using 1.5 or 3 Tesla CMR scanners in LCP patients.

\section{Methods}

Fifteen patients with an LCP (Micra ${ }^{\mathrm{Tm}}$, Medtronic, Minneapolis, USA) implanted at least 6 weeks prior to CMR scan were enrolled in this prospective, single-center, observational study. Patients with other ferromagnetic implanted devices which may interact with the CMR scanner were excluded. The study participants were randomized in a 1:1 ratio into two groups: The study participants underwent CMR imaging in either a 1.5 Tesla CMR scanner (Magnetom Avanto Fit, Siemens Healthineers, Erlangen, Germany) or a 3 Tesla CMR scanner (Magnetom Skyra, Siemens Healthineers) with a maximum gradient field of $45 \mathrm{mT} / \mathrm{m}$ and a slew rate of $200 \mathrm{~T} / \mathrm{m} / \mathrm{s}$. The Micra ${ }^{\mathrm{Tm}} \mathrm{LCP}$ is a MRI conditional single-chamber cardiac pacemaker and the device sizes $25.9 \times 6.7 \mathrm{~mm}$. LCP were interrogated before and immediately after the CMR scan and were programmed to an asynchronous, MRI conditional pacing mode (VOO, $80 \mathrm{bpm}$ ) for the CMR scan. During the CMR scan patients were monitored by continuous electrocardiogram (ECG) and pulse oximetry. Blood pressure measurements were performed before and after CMR scans. An intercom was available for patient communication in the CMR scanners.

The CMR protocol was conducted according to the recommendations of the Society for Cardiovascular Magnetic Resonance (SCMR) [17]. We obtained multiple slice transversal balanced steady-state free precession (bSSFP) images for anatomical orientation and bSSFP cine images in the long axis (4- and 2-chamber view of the left ventricle (LV), LV outflow tract view, 2-chamber view of the right ventricle (RV), RV outflow tract view) and multiple short axis of the LV for function evaluation. A fast low angle shot (FLASH) gradient echo (GRE) based sequence was performed of the 4-chamber view of the LV and T1- and T2-weighted Turbo Spin Echo (TSE) sequences were obtained of the 4-chamber view and in the short axis. CMR sequences and parameters for 1.5 and 3 Tesla CMR scans are shown in Table 1. One patient was excluded from the analysis because of an incomplete CMR investigation due to claustrophobia.

All available CMR images were reviewed by four experienced CMR observers (two cardiologists and two radiologists). In case of inter-observer differences in artefact quantification agreement was reached in case discussion. The LV was divided into 16 segments according to the American Heart Association (AHA) 16 myocardial segmentation classification system [18]. The RV was divided into 3 segments (basal, mid and apical).

LCP related artefacts were graduated as suggested by Klein-Wiele et al. [19]:

Grade 1: excellent image quality, no artefacts affecting myocardial segments or cardiac structures

Grade 2: good image quality with artefact adjacent to the myocardial segments or cardiac structures, delineation of myocardial borders may be limited, no impact on diagnostic value

Grade 3: artefact moderately affecting cardiac structures, less than half of the myocardial segment is superimposed by the artefact

Grade 4: poor image quality with significant artefact affecting more than half of the myocardial segment, non-diagnostic image

The artefact size was measured for every patient on short axis bSSFP cine images which showed the largest artefact. The artefact burden was calculated as affected myocardial segments by grade 1 (excellent), grade 2 (good), grade 3 (moderately) and grade 4 artefacts (poor image quality) divided by 224 (16 myocardial segments $\times 14$ study participants) for the LV and divided by 42 (3 myocardial segments $\times 14$ study participants) for the RV. 


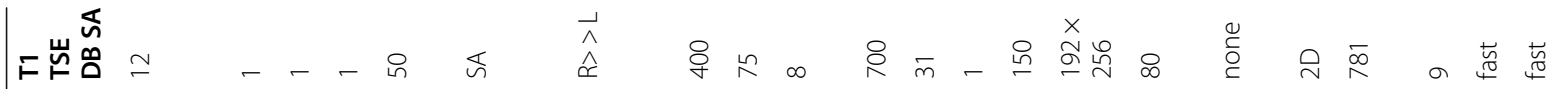
岕守 $F_{0}^{\infty}=\ldots$ - $\frac{\text { h }}{\mathfrak{y}} \hat{x}$

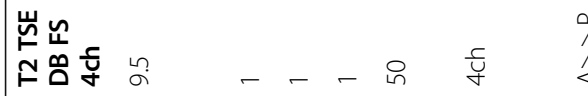

岁号

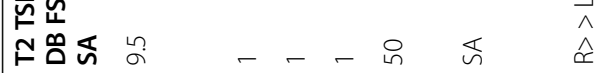

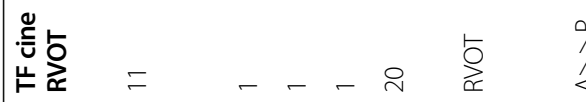

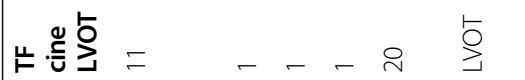

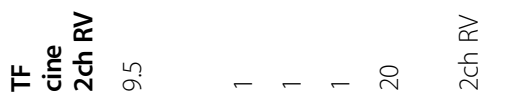

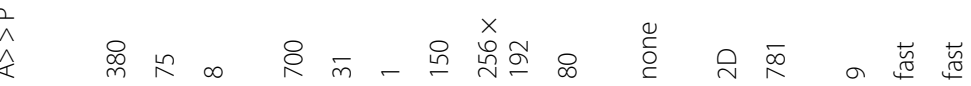

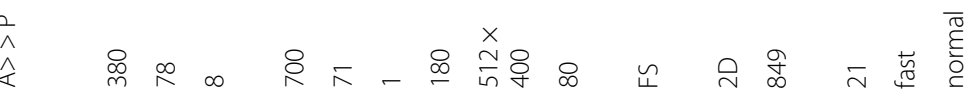

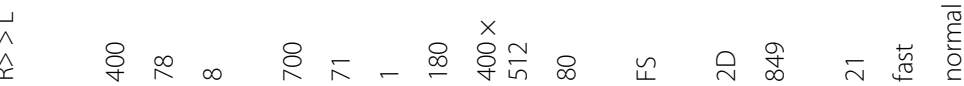

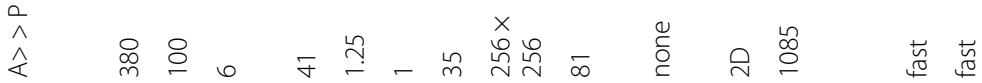

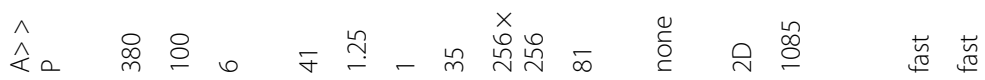

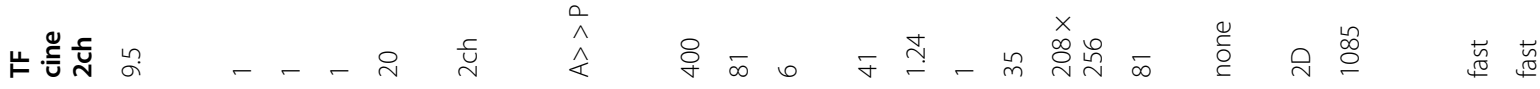

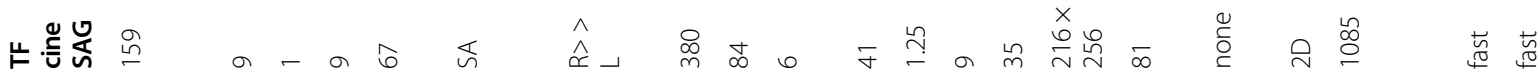

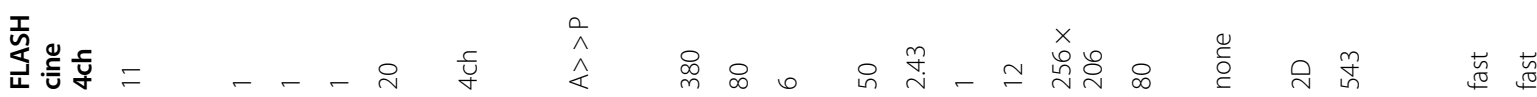
站旁 㞾旁喜

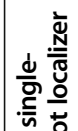

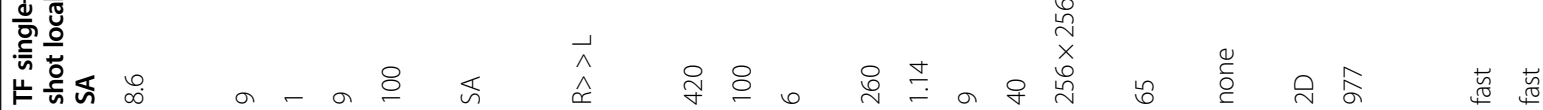
을

药

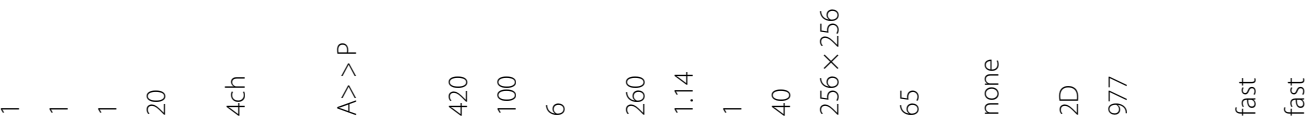

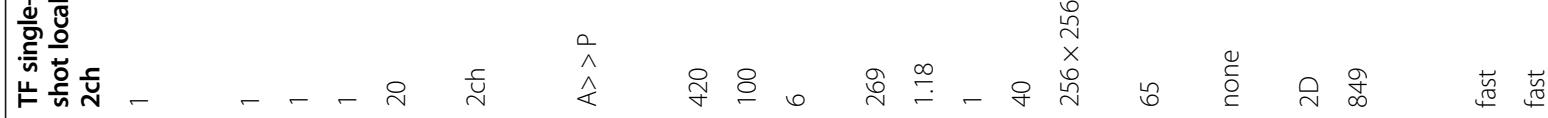

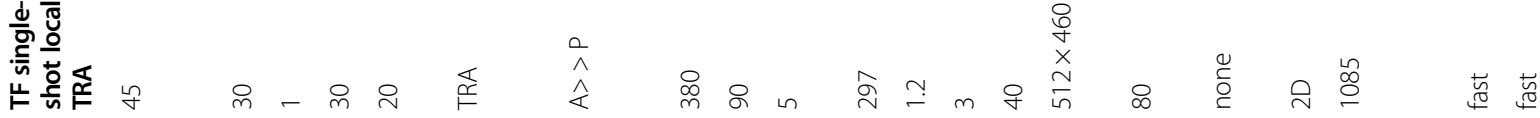

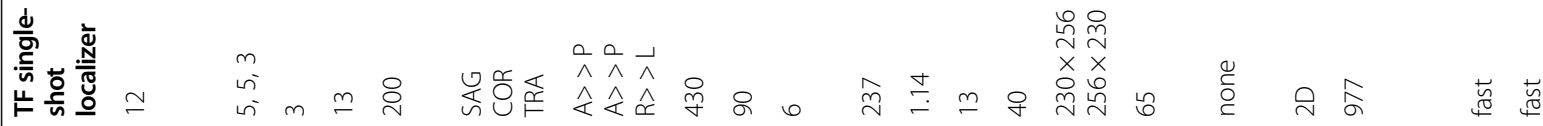

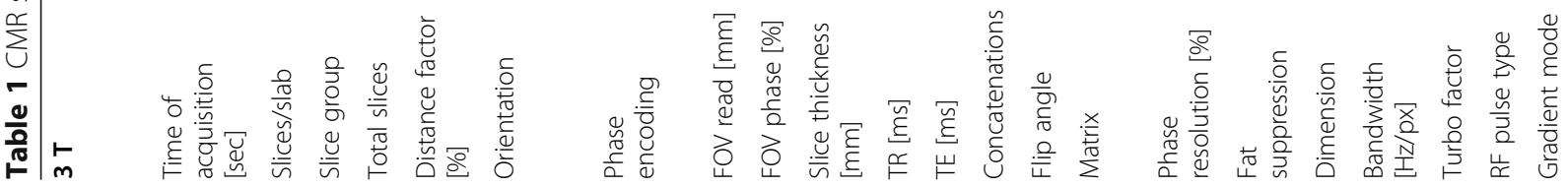


峞毫

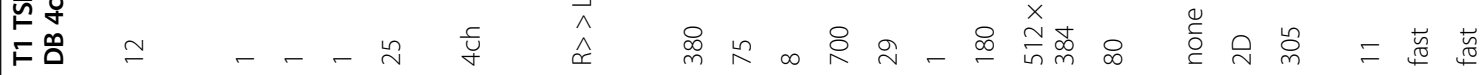

嵌品

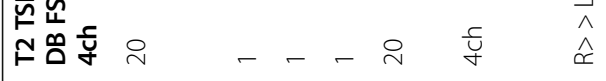

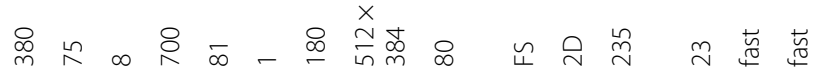

岁氐

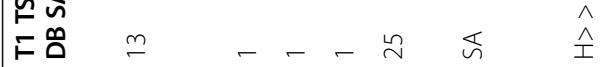

岁号

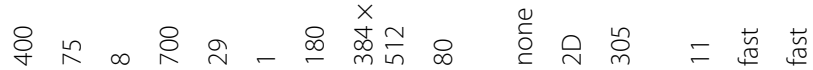

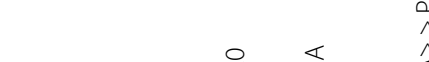

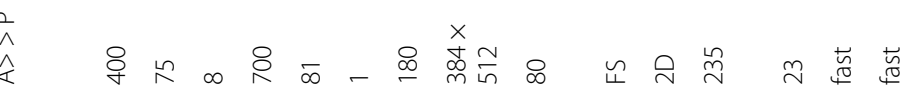

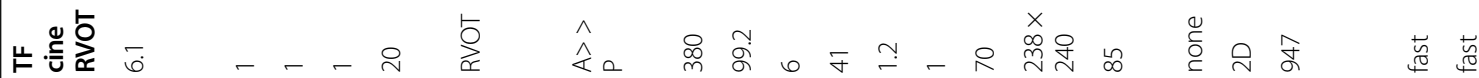

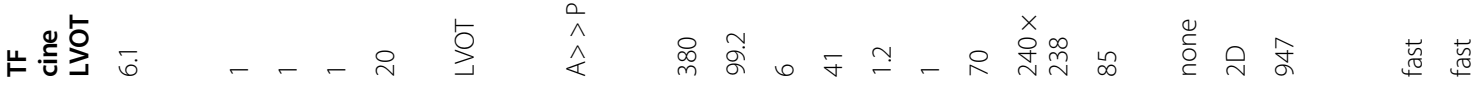

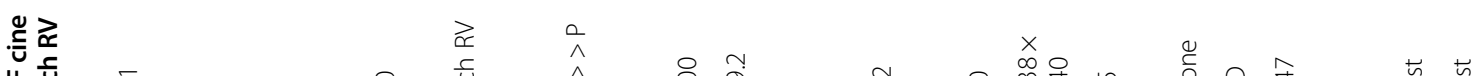

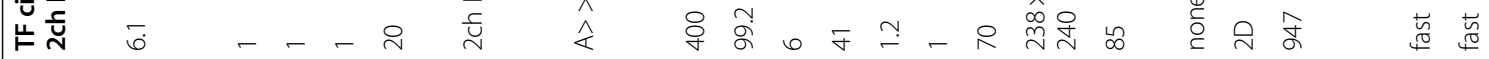

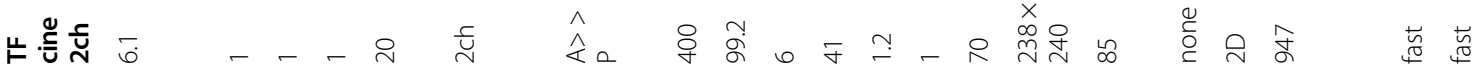

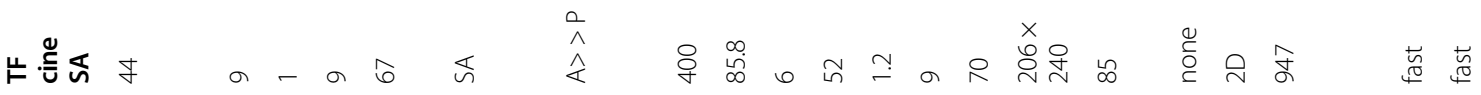

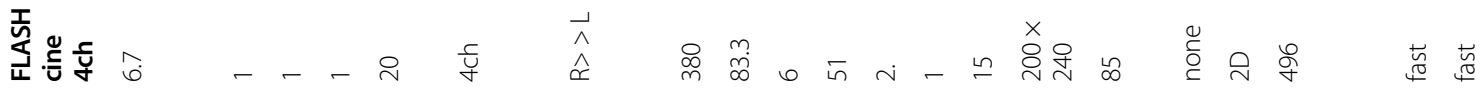

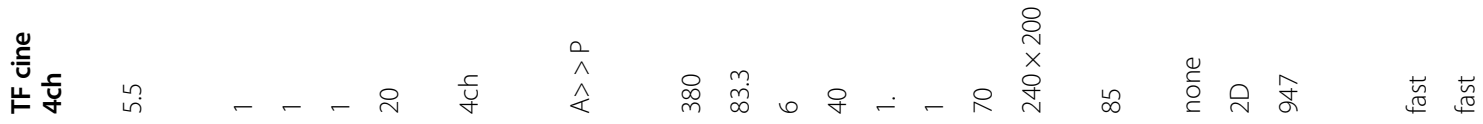

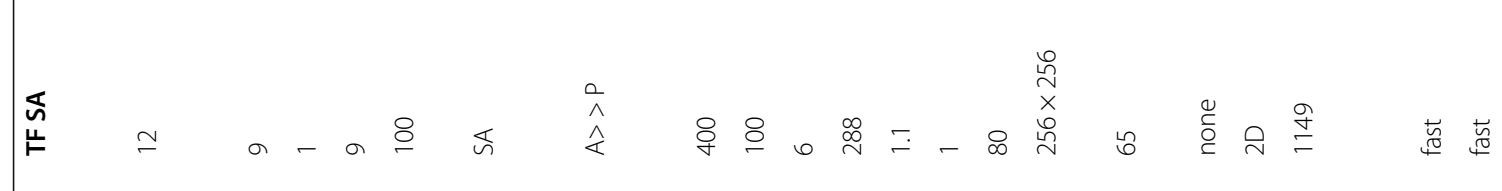

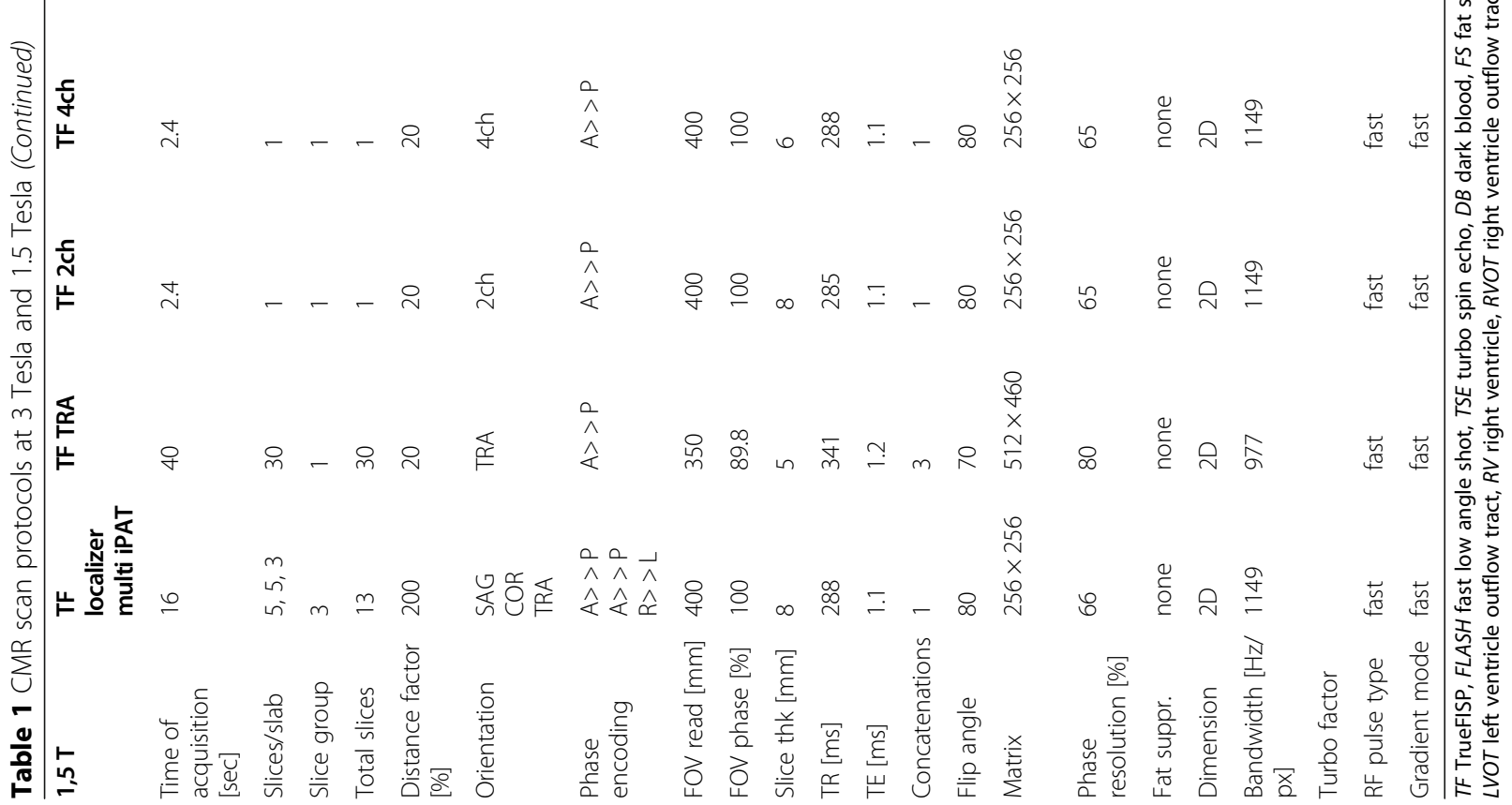


The artefact burden ratio per patient was calculated as affected myocardial segments by grade 3 and 4 artefacts on 1.5 Tesla and 3 Tesla CMR images for the LV and RV.

The study design was approved by the local ethics committee and was conducted according to the Declaration of Helsinki. Written informed consent was obtained from all study participants.

\section{Statistical analysis}

Categorical parameters are described as absolute number and percentage. Continuous values are presented as means \pm standard deviation or means with $95 \%$ confidence intervals $(95 \% \mathrm{CI})$. Differences between groups involving normally distributed data were analyzed by the unpaired $t$ test; those involving not normally distributed data, by the Mann-Whitney $\mathrm{U}$ test; and those involving proportions, by the chi-square test. A two-sided $p$-value $<0.05$ was considered statistically significant. All calculations were performed with SPSS statistical software (Version 21, SPSS Inc., Chicago, Illinois, USA).

\section{Results}

\section{Baseline characteristics}

Fifteen patients were enrolled in this prospective, single-center, observational study. The 1.5 Tesla and 3 Tesla group comprised seven patients each. One study participant was excluded from the study because of an incomplete CMR investigation due to claustrophobia. The remaining 14 patients (female: $n=3$ ) had a mean age of $77.8 \pm$ 14.6 years and all of them had undergone implantation of a LCP at least 6 weeks prior the CMR scan. Indications for pacemaker implantation were bradycardic arrhythmias in permanent atrial fibrillation $(n=11)$ or third degree atrioventricular block $(n=3)$. Baseline characteristics of the study population including comorbidities are shown in Table 2.

\section{Image quality and artefacts}

The LCP caused an arc-shaped artefact at the site of implantation of the RV apex (Fig. 1). Of 224 analyzed myocardial segments of the LV, 158 (70.5\%) were affected by grade $1,27(12.1 \%)$ by grade $2,17(7.6 \%)$ by grade 3 and $22(9.8 \%)$ by grade 4 artefacts. Of 42 analyzed segments of the RV, 20 (47.6\%) were affected by grade $1,6(14.3 \%)$ by grade $2,5(11.9 \%)$ by grade 3 and $11(26.2 \%)$ by grade 4 artefacts. Representative bSSFP cine images of the 4-chamber view with artefact grading obtained with 1.5 Tesla and 3 Tesla are shown in Fig. 1. The artefact area, which was quantified on short axis bSSFP cine images, was slightly higher but not significantly different in the 3 Tesla group compared to the 1.5 Tesla group (both groups: $0.99 \pm 0.16 \mathrm{~cm}^{2}, 3$ Tesla vs 1.5 Tesla: $1.02 \pm 0.19 \mathrm{~cm}^{2}$ vs $0.95 \pm 0.14 \mathrm{~cm}^{2}, p=0.41$ ).
Table 2 Baseline characteristics including indication for pacemaker implantation and comorbidities

\begin{tabular}{ll}
\hline Baseline characteristics $(n=14)$ & 3 female (21.4\%) \\
\hline Sex & $77.8 \pm 14.6$ years \\
Age & \\
Indication for pacemaker implantation & $11(78.6 \%)$ \\
Bradycardiac arrhythmia in atrial fibrillation & $3(21.4 \%)$ \\
3rd degree AV-block & \\
Comorbidities & $12(85.7 \%)$ \\
Atrial fibrillation & $6(42.9 \%)$ \\
Coronary artery disease & $8(57.1 \%)$ \\
Hypertension & $3(21.4 \%)$ \\
Diabetes & $6(42.9 \%)$ \\
Chronic kidney disease & $2(14.3 \%)$ \\
Peripheral artery disease & $2(14.3 \%)$ \\
History of stroke &
\end{tabular}

The artefact burden ratio per patient of affected myocardial segments by grade 3 and 4 artefacts on the short axis bSSFP cine images of the LV was significantly higher in the 3 Tesla group ( 3 Tesla vs 1.5 Tesla: $3.7 \pm 1.6$ vs $1.9 \pm$ 1.4 myocardial segments per patient, $p=0.03$ ). The detailed analysis of myocardial segments affected by grade 3 and grade 4 artefacts revealed a high artefact burden particularly in the mid anteroseptal, inferoseptal and apical septal myocardial segments (AHA myocardial segments 8 , 9,14 ) with an artefact burden of 50,50 and $85.7 \%$ in these regions, respectively. These artefacts were more pronounced in patients undergoing CMR on 3 Tesla CMR scanners ( 3 Tesla vs 1.5 Tesla: $57.1 \%$ vs $42.9 \%$. $57.1 \%$ vs $42.9,100 \%$ vs $71.4 \%)$. There were no artefacts in the basal inferoseptal, inferior, basal to apical anterolateral and inferolateral myocardial segments (AHA myocardial segments 3, 4, 5, 6, 11, 12 and 16). Details of artefact distribution in the short axis are demonstrated in Fig. 2 and Table 3.

Quantification of LV function and volumetry were feasible in 14 patients (100\%) with a mean LV ejection fraction of $49 \pm 7.4 \%$. We experienced more problems of ECG-triggering in patients undergoing 3 Tesla CMR scans (3 Tesla vs 1.5 Tesla: 3 vs 0 patients). In one 3 Tesla patient, pulse-triggering was necessary for image acquisition. Analysis of the RV was evenly impaired in both cohorts due to artefacts of the LCP severely compromising the image quality of particularly the mid and apical free wall of the RV (grade 3 and 4 artefacts RV basal: 0\%, mid: $21.4 \%$, apical: $92.9 \%$ ). The artefact burden ratio of affected myocardial segments by grade 3 and 4 artefacts on short axis bSSFP cine images of the RV revealed $1.1 \pm 0.4$ segments per patient. Therefore, an exact quantification of 


Fig. 1 Balanced steady free precession (bSSFP) cine images of the 4 chamber view with grade 1 , grade 2, grade 3 and grade 4 artefacts of the
apical septal myocardial segment obtained with 1.5 Tesla and 3 Tesla. No patient in the 3 Tesla group showed a grade 1 artefact of the apical
septal myocardial segment. * artefact caused by the leadless cardiac pacemaker (LCP)

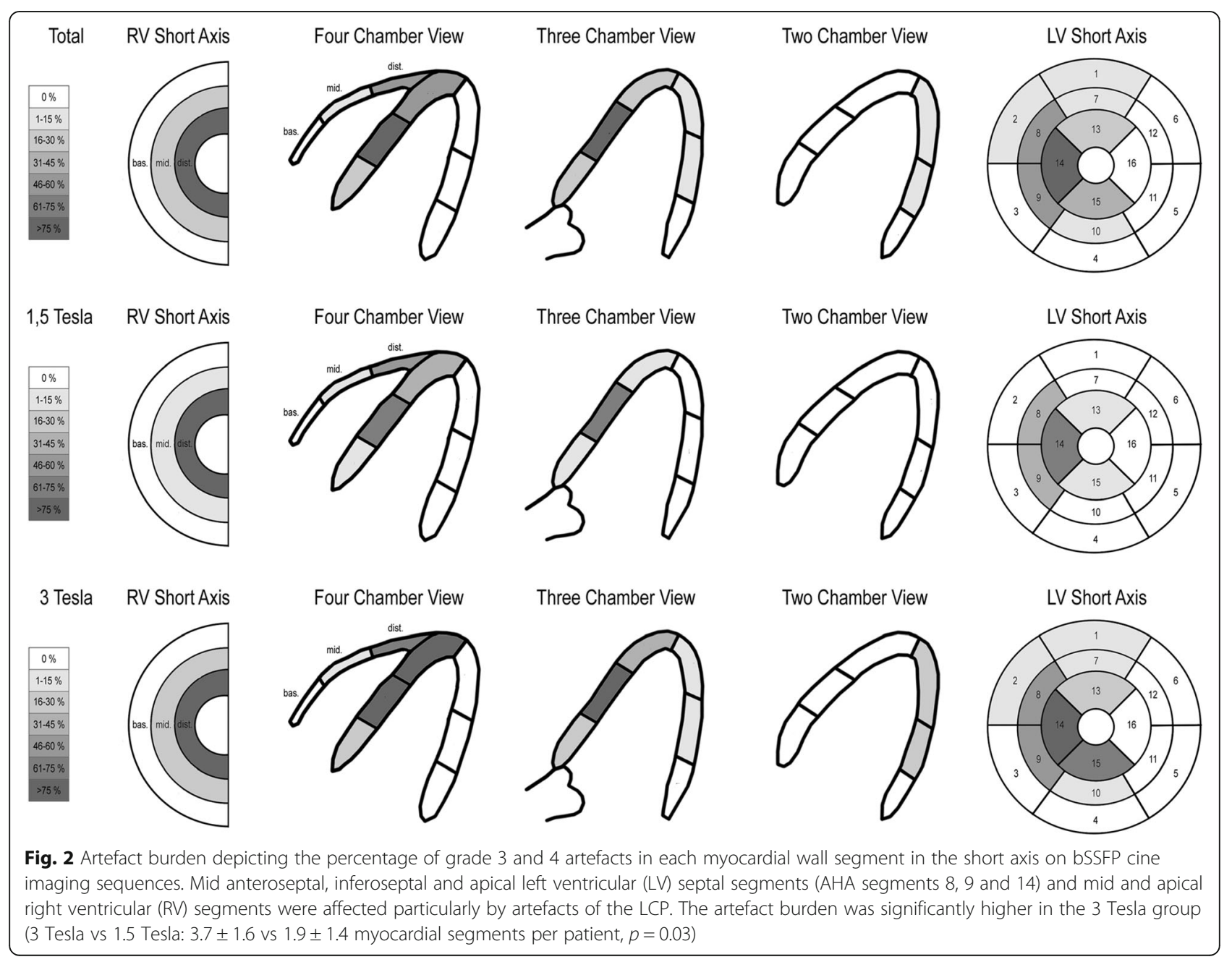


Table 3 Artefact burden of myocardial segments of the LV and RV according to the AHA myocardial segmentation system in the short axis $(n=14)$

\begin{tabular}{|c|c|c|c|c|c|c|c|c|c|c|c|c|c|c|c|c|c|c|c|}
\hline \multirow{2}{*}{$\frac{\text { All [\%] }}{\text { Artefact }}$} & \multicolumn{6}{|l|}{ Basal } & \multicolumn{6}{|l|}{ Mid } & \multicolumn{4}{|c|}{ Apical } & \multicolumn{3}{|l|}{ RV } \\
\hline & 1 & 2 & 3 & 4 & 5 & 6 & 7 & 8 & 9 & 10 & 11 & 12 & 13 & 14 & 15 & 16 & basal & mid & apical \\
\hline grade 1 & 92.9 & 78.6 & 85.7 & 100 & 100 & 100 & 64.3 & 21.4 & 21.4 & 78.6 & 100 & 100 & 35.7 & 7.1 & 42.9 & 100 & 100 & 42.9 & 0 \\
\hline grade 2 & 0 & 14.3 & 14.3 & 0 & 0 & 0 & 28.6 & 28.6 & 28.6 & 14.3 & 0 & 0 & 42.9 & 7.1 & 14.3 & 0 & 0 & 35.7 & 7.1 \\
\hline grade 3 & 7.1 & 0 & 0 & 0 & 0 & 0 & 7.1 & 7.1 & 14.3 & 7.1 & 0 & 0 & 21.4 & 14.3 & 42.9 & 0 & 0 & 14.3 & 21.4 \\
\hline grade 4 & 0 & 7.1 & 0 & 0 & 0 & 0 & 0 & 42.9 & 35.7 & 0 & 0 & 0 & 0 & 71.4 & 0 & 0 & 0 & 7.1 & 71.4 \\
\hline $3 T$ & 1 & 2 & 3 & 4 & 5 & 6 & 7 & 8 & 9 & 10 & 11 & 12 & 13 & 14 & 15 & 16 & basal & mid & apical \\
\hline grade 1 & 85.7 & 71.4 & 85.7 & 100 & 100 & 100 & 57.1 & 0 & 14.3 & 57.1 & 100 & 100 & 0 & 0 & 14.3 & 100 & 100 & 42.9 & 0 \\
\hline grade 2 & 0 & 14.3 & 14.3 & 0 & 0 & 0 & 28.6 & 42.9 & 28.6 & 28.6 & 0 & 0 & 71.4 & 0 & 14.3 & 0 & 0 & 28.6 & 0 \\
\hline grade 3 & 14.3 & 0 & 0 & 0 & 0 & 0 & 14.3 & 14.3 & 14.3 & 14.3 & 0 & 0 & 28.6 & 0 & 71.4 & 0 & 0 & 28.6 & 0 \\
\hline grade 4 & 0 & 14.3 & 0 & 0 & 0 & 0 & 0 & 42.9 & 42.9 & 0 & 0 & 0 & 0 & 100 & 0 & 0 & 0 & 0 & 100 \\
\hline $1.5 \mathrm{~T}$ & 1 & 2 & 3 & 4 & 5 & 6 & 7 & 8 & 9 & 10 & 11 & 12 & 13 & 14 & 15 & 16 & basal & mid & apical \\
\hline grade 1 & 100 & 85.7 & 85.7 & 100 & 100 & 100 & 71.4 & 42.9 & 71.4 & 100 & 100 & 100 & 71.4 & 14.3 & 71.4 & 100 & 100 & 42.9 & 0 \\
\hline grade 2 & 0 & 14.3 & 14.3 & 0 & 0 & 0 & 28.6 & 14.3 & 0 & 0 & 0 & 0 & 14.3 & 14.3 & 14.3 & 0 & 0 & 42.9 & 14.3 \\
\hline grade 3 & 0 & 0 & 0 & 0 & 0 & 0 & 0 & 0 & 28.6 & 0 & 0 & 0 & 14.3 & 28.6 & 14.3 & 0 & 0 & 0 & 42.9 \\
\hline grade 4 & 0 & 0 & 0 & 0 & 0 & 0 & 0 & 42.9 & 0 & 0 & 0 & 0 & 0 & 42.9 & 0 & 0 & 0 & 14.3 & 42.9 \\
\hline
\end{tabular}

RV function was not possible in both groups and the RV function was only assessed visually (normal: $n=7$, mildly impaired: $n=6$, moderately impaired: $n=0$, severely impaired: $n=1$ ).

The evaluation of T1 and T2 weighted images showed better image quality with smaller arc-shaped artefacts compared to bSSFP cine imaging and less grade 3 and 4 artefacts in the mid anteroseptal, inferoseptal and septal apical myocardial segments (Figs. 3 and 4). Furthermore, when comparing 4-chamber view bSSFP cine images and GRE based FLASH cine images, there was only a slightly better image quality on FLASH sequences in the mid and apical septal myocardial segments of the LV (Fig. 3).

Aortic, mitral and tricuspid valves could be assessed in all patients (grade 3 and 4 artefacts: 0, 0, 0\%, respectively). We did not observe any hemodynamically relevant tricuspid valve insufficiency in our study cohort of patients with LCP.

\section{Safety and device integrity}

There were no clinical or device-related serious adverse events during CMR scans. The CMR scans, both 1.5 and 3 Tesla, did not lead to malfunction of the implanted LCP devices. We observed no significant change of pacing thresholds (before CMR scan: $0.59 \pm 0.15 \mathrm{~V} /$ $0.24 \mathrm{~ms}$, after: $0.61 \pm 0.17 \mathrm{~V} / 0.24 \mathrm{~ms}, p=0.08$ ) and a marginal, but statistically significant increase of sensing amplitude (before CMR scan: $14.9 \pm 4.7 \mathrm{mV}$, after: 15.8 $\pm 4.5 \mathrm{mV}, p=0.02$ ) and impedances (before CMR scan: $527 \pm 100 \Omega, \quad$ after: $541 \pm 110 \Omega, \quad \mathrm{p}=0.02)$. Battery voltage remained stable (before CMR scan: > 10 years, after: $>10$ years).

\section{Discussion}

This prospective, single-center, observational study, demonstrates that CMR imaging in patients with LCP implanted at least 6 weeks prior to the CMR scan is feasible. Overall image quality was excellent or good in the majority of CMR images (myocardial segments of the LV affected by grade 1: $70.5 \%$, grade $2: 12.1 \%$, grade 3: $7.6 \%$, grade $4: 9.8 \%)$. Artefacts occurred particularly in the mid anteroseptal, inferoseptal and septal apical myocardial segments (AHA myocardial segments 8, 9 and 14) and in the mid and apical segments of the RV. 3 Tesla CMR imaging led to a significantly higher artefact burden ratio per patient compared to 1.5 Tesla CMR imaging. Assessment of LV function and aortic, mitral and tricuspid valve, as well as tissue characterization by $\mathrm{T} 1$ - and $\mathrm{T} 2$ - weighted imaging was feasible with both modalities.

Several studies have demonstrated the safety and feasibility of MRI conditional, transvenous pacemakers and ICD undergoing MRI [3-11]. Potential adverse effects of MRI on implanted cardiac devices include: radiofrequency-induced heating of the lead tips, pacing inhibition/dysfunction, asynchronous pacing with the possibility of induction of ventricular tachyarrhythmias, change or loss of programmed data and changes in capture treshold [2]. A closer distance of the scanning area to the pacing system and a higher field strength increases this risk [2]. Therefore, pacemakers and ICD 

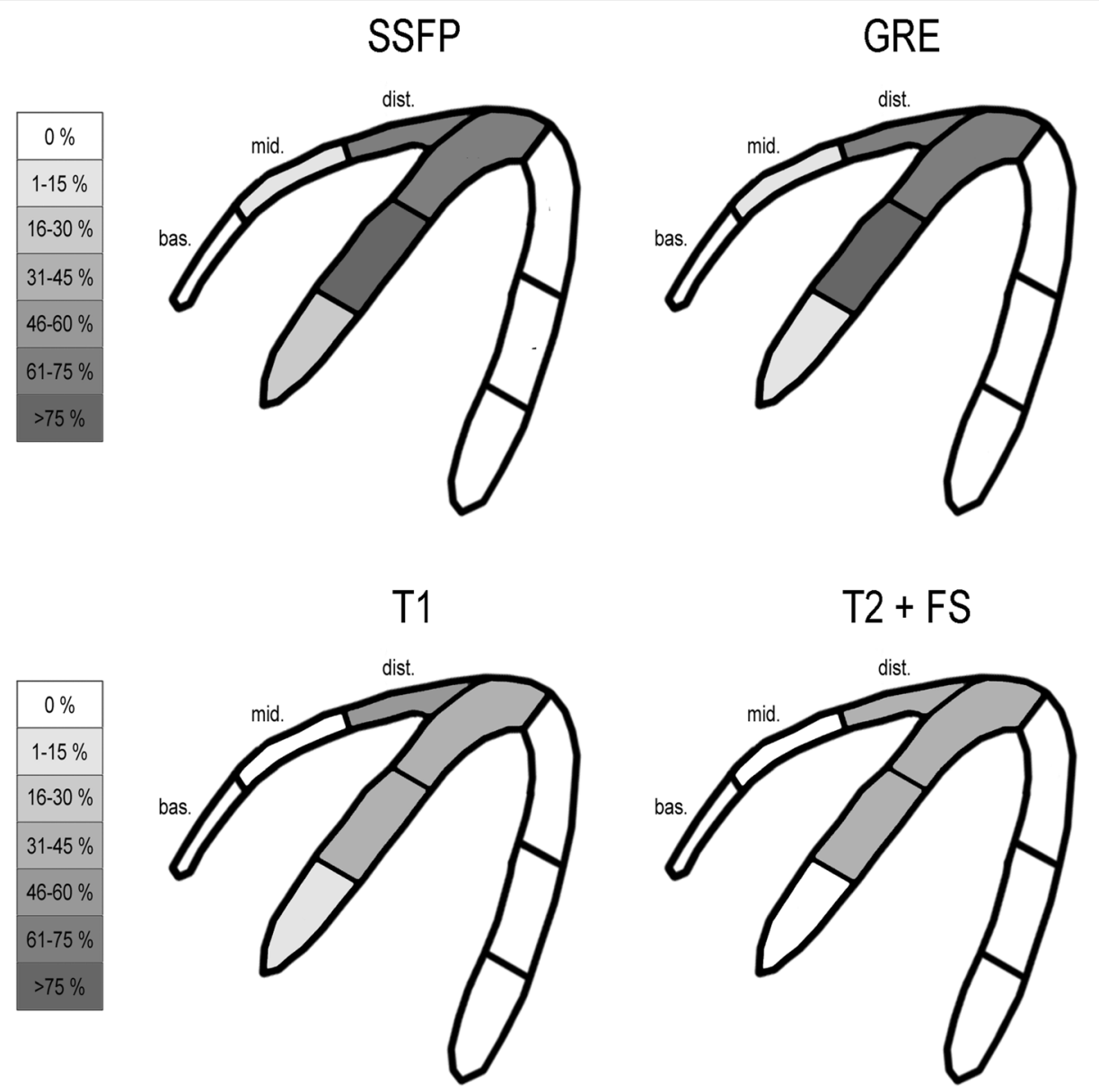

Fig. 3 Artefact burden of grade 3 and 4 artefacts of the 4 chamber view with different CMR sequences showed better image quality on T1 and T2 weighted images and only slightly less artefacts on gradient recalled echo (GRE) based fast low angle shot (FLASH) images compared to bSSFP images
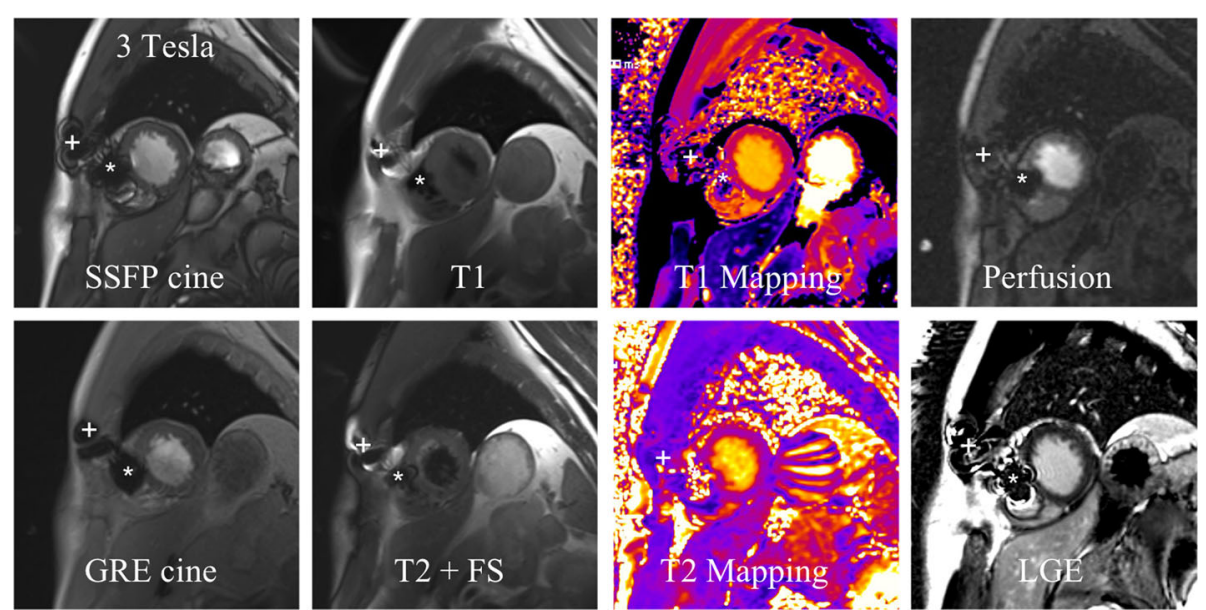

Fig. 4 Artefacts due to the LCP in one patient with different CMR sequences obtained with 3 Tesla in short axis orientation showing slightly more artefacts on bSSFP cine images compared to GRE cine, T1, T2 with fat saturation, $\mathrm{T} 1$ and $\mathrm{T} 2$ mapping, perfusion and LGE images. * $=$ artefact caused by the LCP, $+=$ artefact caused by implantable loop recorder 
including leads must be interrogated before and after MRI scans and have to be programmed to MRI conditional pacing modes during the scan. It is recommended to monitor patients with cardiac devices with ECG, pulse oximetry and blood pressure measurements during MRI scans [2]. Soejima et al. demonstrated in an ex-vivo study the safety for the Micra ${ }^{\mathrm{ma}}$ LCP in a not perfused phantom model with a device heating of less than $0.4{ }^{\circ} \mathrm{C}$ at 1.5 Tesla and $0.5{ }^{\circ} \mathrm{C}$ at 3 Tesla MRI [20]. Furthermore they report no MRI-related complication in a clinical case study. We did not observe any clinical or device-related serious adverse events in our study cohort. Pacing threshold did not change significantly and battery voltage remained stable before and after CMR scans. However, we observed a marginal, but statistically significant increase of sensing amplitude (before CMR scan: $14.9 \pm 4.7 \mathrm{mV}$, after: $15.8 \pm 4.5 \mathrm{mV}, p=0.02)$ and impedances (before CMR scan: $527 \pm 100 \Omega$, after: $541 \pm$ $110 \Omega, \mathrm{p}=0.02$ ). We do not consider these marginal changes to be clinically relevant because we did not observe any malfunction of LCP during or after CMR scans. Further studies are needed to provide follow up data of LCP after MRI.

Pacemakers and other implanted cardiac electronic devices lead to metallic susceptibility artefacts due to distortion of the magnetic field [15]. Artefacts tend to be larger on 3 Tesla CMR scanners which could be confirmed by our study findings with a higher artefact burden of affected myocardial segments of the LV by grade 3 and 4 artefacts in patients undergoing CMR imaging at 3 Tesla ( 3 Tesla vs 1.5 Tesla: $3.7 \pm 1.6$ vs $1.9 \pm$ 1.4 AHA myocardial segments, $p=0.03$ ). These susceptibility artefacts were pronounced in particular in the mid anteroseptal, inferoseptal and apical septal AHA myocardial segments 8,9 and 14 of the LV and were even more pronounced in patients undergoing CMR on 3 Tesla. The RV was mainly affected in the mid and apical myocardial segments. The size of the arc-shaped artefact by the LCP $\left(0.99 \pm 0.16 \mathrm{~cm}^{2}\right)$ was not significantly different in both groups ( 3 Tesla vs 1.5 Tesla: $1.02 \pm 0.19 \mathrm{~cm}^{2}$ vs $\left.0.95 \pm 0.14 \mathrm{~cm}^{2}, p=0.41\right)$. The size of the area affected by the artefact was 7 -fold larger compared to the size of the LCP device itself. Quantification of LV function and volumetry were feasible in all patients $(n=14)$. However, the RV function could only be assessed visually, because of the high artefact burden, demonstrating 7 patients with normal, 6 patients with mildly and 1 patient with severely reduced RV function. However, the impaired RV function cannot be interpreted as a reduced RV function due to the LCP because we did not compare RV function before and after implantation of the LCP.

As described above, mid anteroseptal, inferoseptal and apical septal myocardial segments of the LV and mid and apical segments of the RV were affected by artefacts of the LCP in the majority of patients which may impair or even exclude diagnostic evaluation of these segments especially on perfusion and late gadolinum enhancement images. In contrast to our findings, Klein-Wiele et al. revealed in a population of 61 patients with MRI conditional, transvenous pacemakers no relevant artefacts in patients with right-sided devices irrespective of the imaging sequence [19]. There were no pacemaker induced artefacts in left-sided implants in first pass perfusion sequence, flow analysis and T1 weighted imaging. bSSFP cine sequences tend to have more artefact burden than late gadolinum enhancement sequences [19]. As reported by Sasaki et al. right-sided pacemakers and ICD did not cause susceptibility artefacts on CMR images but artefacts of the anterior and apical LV were described with left-sided ICD [21]. To the best of our knowledge, there are no prospective studies investigating artefacts on CMR scans in patients with LCP. We recently reported about a case of a patient with a LCP and an arc-shaped artefact at the RV apex [16]. As described by Klein-Wiele et al., pacing leads artefacts are smaller and do not usually interfere with myocardial structures which may be a potential advantage of transvenous cardiac pacemaker systems undergoing CMR imaging [19].

LCP are RV single-chamber pacemakers which are implanted by using a femoral percutaneous approach [12]. The next step of leadless technology will be dual-chamber pacing to treat patients with AV block [13]. Aurrichio et al. reported about feasibility, safety and short-term outcome of leadless ultrasound-based endocardial LV resychronization in patients with heart failure [22]. A study by Tjong FV et al. reported about leadless pacing combined with subcutaneous defibrillation therapy [23]. Therefore, the clinical use of these leadless cardiac devices will increase in the next years. Keller $J$ et al. reported artefacts by the can of subcutaneous ICD affecting the LV [24]. This is consistent with our expierence in this patient undergoing CMR imaging that diagnostic evaluation of the LV is severely impaired by artefacts of the subcutaneous ICD.

CMR is an important non-invasive imaging tool to assess patients with cardiovascular diseases by evaluating myocardial function, wall motion abnormalities, viability, coronary perfusion, valves and tissue characterization [1]. Therefore, we were able to demonstrate that CMR is feasible in patients with LCP. Furthermore, the overall image quality was excellent to good and the artefact burden due to the LCP was comparable small and allowed a comprehensive evaluation of the cardiac structures and function, wall motion abnormalities and tissue characterization. However, mid anteroseptal, inferoseptal and apical septal myocardial segments of the LV were affected by artefacts due to the LCP which may impair or even exclude diagnostic evaluation of these segments, 
especially on perfusion and LGE images (Fig. 4). The artefact burden on CMR images could be significantly reduced by the use of 1.5 Tesla MRI scanners.

\section{Limitations of this study}

Besides the limited number of patients this study has further limitations. This study enrolled only patients with Micra $^{\mathrm{ma}}$ LCP and the described artefacts are specific for this LCP. Further studies are needed to evaluate the artefact burden of LCP from other manufactures. However, according to our data the size of the artefact by the LCP did not differ widely with only a small standard deviation on CMR images $\left(0.99 \pm 0.16 \mathrm{~cm}^{2}\right)$. Therefore, we conclude that the localization of the artefact depends mainly on the site of implantation of the LCP which is usually at the apex of the RV or at the apical or mid right interventricular septum. The impaired RV function in 7 patients of our study population cannot be interpreted as a reduced RV function due to the LCP because we did not compare RV function before and after implantation of the LCP. Furthermore, we were not able to report on late gadolinum enhancement sequences as these sequences were not part of our CMR scan protocol. As reported by Klein-Wiele, late gadolinum enhancement sequences showed a lower artefact burden compared to bSSFP cine images [19].

\section{Conclusion}

This prospective, single-center, observational study demonstrates that CMR imaging in patients with LCP implanted at least six prior to the CMR scan is feasible. Overall image quality was excellent to good in the majority of CMR images. Assessment of LV function and aortic, mitral and tricuspid valve as well as tissue characterization with T1 and T2 weighted imaging were feasible with 1.5 Tesla and 3 Tesla. However, mid anteroseptal, inferoseptal and apical septal myocardial segments of the LV were affected by artefacts of the LCP in the majority of patients which may impair or even exclude diagnostic evaluation of these segments. Artefact burden on CMR images may be reduced by the use of 1.5 Tesla scanners.

\section{Abbreviations}

AHA: American Heart Association; bSSFP: Balanced steady-state free precession; CMR: Cardiovascular magnetic resonance; ECG: Electrocardiogram; FLASH: Fast low angle shot; GRE: Gradient echo; ICD: Implantable cardioverter defibrillators: LCP: Leadless cardiac pacemakers; LV: Left ventricle/left ventricular; MRI: Magnetic resonance imaging; RV: Right ventricle/right ventricular; TSE: Turbo spin echo

\section{Acknowledgements}

The authors want to thank Alexander Kypta MD for supporting this study and, Thomas Lukas MD, Walter Aufreiter and the dedicated staff of the MRI unit of the Kepler University Hospital for image acquisition.

\section{Availability of data and materials}

The datasets used and/or analyzed during the current study are available from the corresponding author on reasonable request.

\section{Authors' contributions}

DK, CR, JK, HB and CS conceived the study design. DK, CR, PS and FF obtained CMR images and analyzed CMR scans. JK, HB and JK interrogated LCP before and after CMR scans. DK, CR, JK and HB analyzed the data. DK drafted the manuscript. As authors, we attest to each of our substantial contributions to the manuscript and critical revision. All authors read and approved the final manuscript.

\section{Ethics approval and consent to participate}

The study design was approved by the local ethics committee (Ethikkommission Land Oberösterreich, Kepler University Hospital Linz, B-123-16) and was conducted according to the Declaration of Helsinki. Written informed consent was obtained from all study participants.

\section{Competing interests}

CS received speakers' honoraria from Medtronic. DK, CR, JK, PS, JK, HB and FF declare no competing interests.

\section{Publisher's Note}

Springer Nature remains neutral with regard to jurisdictional claims in published maps and institutional affiliations.

\section{Author details}

'Department of Cardiology, Kepler University Hospital Linz, Med Campus III, Krankenhausstraße 9, 4021 Linz, Austria. ${ }^{2}$ Department of Radiology, Kepler University Hospital Linz, Linz, Austria. ${ }^{3}$ Medical Faculty of the Friedrich Alexander University of Erlangen-Nürnberg, Erlangen, Germany. ${ }^{4}$ Department of Internal Medicine II, Paracelsus Medical University Salzburg, Salzburg, Austria.

Received: 26 November 2017 Accepted: 6 June 2018

Published online: 05 July 2018

References

1. Penell DJ. Cardiovascular magnetic resonance. Circulation. 2010;121(5):692-705

2. Brignole $M$, Auricchio A, Baron-Esquivias $G$, Bordachar P, Boriani $G$, Breithardt OA, et al. 2013 ESC guidelines on cardiac pacing and cardiac resynchronization therapy. Europace. 2013;15(8):1070-118.

3. Kalb B, Indik JH, Ott P, Martin DR. MRI of patients with implanted cardiac devices. J Magn Reson Imaging. 2017; https://doi.org/10.1002/jmri.25824.

4. Russo RJ, Costa HS, Silva PD, Anderson JL, Arshad A, Biederman RW, et al. Assessing the risks associated with $\mathrm{MRI}$ in patients with a pacemaker or defibrillator. N Engl J Med. 2017;376(8):755-64.

5. Strom JB, Whelan JB, Shen C, Zheng SQ, Mortele KJ, Kramer DB. Safety and utility of magnetic resonance imaging in patients with cardiac implantable electronic devices. Heart Rhythm. 2017;14(8):1138-44.

6. Shah AD, Patel AU, Knezevic A, Hoskins MH, Hirsh DS, Merchant FM, et al. Clinical performance of magnetic resonance imaging conditional and nonconditional cardiac implantable electronic devices. Pacing Clin Electrophysiol. 2017;40(5):467-75.

7. Bertelsen L, Petersen HH, Philbert BT, Svendsen JH, Thomsen C, Vejlstrup N. Safety of magnetic resonance scanning without monitoring of patients with pacemakers. Europace. 2017;19(5):818-23.

8. Camacho JC, Moreno CC, Shah AD, Mittal PK, Mengistu A, Lloyd MS, et al. Safety and quality of 1.5-T MRI in patients with conventional and MRIconditional cardiac implantable electronic devices after implementation of a standardized protocol. AJR Am J Roentgenol. 2016;207(3):599-604.

9. Horwood L, Attili A, Luba F, Ibrahim EH, Parmar H, Stojanovska J, et al. Magnetic resonance imaging in patients with cardiac implanted electronic devices: focus on contraindications to magnetic resonance imaging protocols. Europace. 2017;19(5):812-7.

10. Lowe MD, Plummer CJ, Mainsty CH, Linker NJ, British Heart Rhythm Society. Safe use of MRI in people with cardiac implantable electronic devices. Heart. 2015;101(24):1950-3.

11. Kypta A, Blessberger H, Hoenig S, Saleh K, Lambert T, Kammler J, et al. Clinical safety of an MRI conditional implantable cardioverter defibrillator system: a prospective monocenter ICD-magnetic resonance imaging feasibility study (MIMI). J Magn Reson Imaging. 2016;43(3):574-84.

12. Tjong FV, Reddy W. Permanent leadless cardiac pacemaker therapy: a comprehensive review. Circulation. 2017;135(15):1458-70. 
13. Kiehl EL, Cantillon DJ. Leadless cardiac pacing: what primary care providers and non-EP cardiologists should know. Cleve Clin J Med. 2016;83(11 Suppl 2):S24-34.

14. Sperzel J, Burri H, Gras D, Tjong FV, Knops RE, Hindricks G, et al. State of the art of leadless pacing. Europace. 2015;17(10):1508-13.

15. Ferreira PF, Gatehouse PD, Mohiaddin RH, Firmin DN. Cardiovascular magnetic resonance artefacts. J Cardiovasc Magn Reson. 2013;15:41.

16. Kypta A, Blessberger H, Kiblboeck D, Steinwender C. Three tesla cardiac magnetic resonance imaging in a patient with a leadless cardiac pacemaker system. Eur Heart J. 2017;38(34):2628.

17. Kramer CM, Barkhausen J, Flamm SD, Kim RJ, Nagel E. Society of Cardiovascular Magnetic Resonance Board of trustees task force on standardized protocols. Standardized cardiovascular magnetic resonance (CMR) protocols 2013 update. J Cardiovasc Magn Reson. 2013;15:91.

18. Cerqueira MD, Weissman NJ, Dilsizian V, Jacobs AK, Kaul S, Laskey WK, et al. Standardized myocardial segmentation and nomenclature for tomographic imaging of the heart. Circulation. 2002;105:539-42.

19. Klein-Wiele O, Garmer M, Busch M, Mateiescu S, Urbien R, Barbone G, et al. Cardiovascular magnetic resonance in patients with magnetic resonance conditional pacemaker systems at 1.5T: influence of pacemaker related artefacts on image quality including first pass perfusion, aortic and mitral valve assessment, flow measurement, short tau inversion recovery and T1-weighted imaging. Int J Cardiovasc Imaging. 2017;33(3):383-94.

20. Soejima K, Edmonson J, Ellingson ML, Herberg B, Wiklund C, Zhao J. Safety evaluation of a leadless pacemaker for magnetic resonance imaging use. Heart Rhythm. 2016:13(10):2056-63.

21. Sasaki T, Hansford R, Zviman MM, Kolandaivelu A, Bluemke DA, Berger RD, et al. Quantitative assessment of artifacts on cardiac magnetic resonance imaging of patients with pacemakers and implantable cardioverterdefibrillators. Circ Cardiovasc Imaging. 2011;4:662-70.

22. Auricchio A, Delnoy PP, Butter C, Brachmann J, Van Erven L, Spitzer S, et al. Feasibility, safety, and short-term outcome of leadless ultrasound-based endocardial left ventricular resynchronization in heart failure patients: results of the wireless stimulation endocardially for CRT (WiSE-CRT) study. Europace. 2014;16(5):681-8.

23. Tjong FV, Brouwer TF, Smeding L, Kooiman KM, de Groot JR, Ligon D, et al. Combined leadless pacemaker and subcutaneous implantable defibrillator therapy: feasibility, safety, and performance. Europace. 2016;18(11):1740-7.

24. Keller J, Neuzil P, Vymazal J, Janotka M, Brada J, Zacek R, et al. Magnetic resonance imaging in patients with subcutaneous implantable cardioverterdefibrillator. Europace. 2015;17(5):761-6.

\section{Ready to submit your research? Choose BMC and benefit from:}

- fast, convenient online submission

- thorough peer review by experienced researchers in your field

- rapid publication on acceptance

- support for research data, including large and complex data types

- gold Open Access which fosters wider collaboration and increased citations - maximum visibility for your research: over $100 \mathrm{M}$ website views per year

At BMC, research is always in progress.

Learn more biomedcentral.com/submissions 